\title{
CUSTOMER CENTRICITY IN TOMORROW'S WORLD HOW 5G WILL TRANSFORM CUSTOMER SERVICE
}

\author{
Swapnil Saurav ${ }^{1}$ \\ ${ }^{1}$ JDA Software, Value Engineering Team, Hyderabad, India
}

\begin{abstract}
$5 G$ offers enormous potential for both consumers and industry As well as the prospect of being considerably faster than existing technologies, $5 G$ holds the promise of applications with high social and economic value, leading to a 'hyperconnected society' in which mobile will play an ever more important role in people's lives. The technical requirements that necessitate a true generational shift are sub-1ms latency and >1 Gbps downlink speed. Achieving sub-1ms latency is a hugely exciting challenge that will define $5 G$.

In this paper, I explore how technology is changing consumer dynamics and show how businesses have an opportunity to influence persistency, retention and expectations through improved customer engagement. I discuss the strategic paradigm by proposing a framework and discussed about the operational areas where the businesses can become more customer focused: developing customer-centric models, applying advanced segmentation and data analytics to design an effective customer experience, harnessing the power of digital in an integrated channel strategy, and shifting to a new culture of innovation that will drive growth and competitive advantage.
\end{abstract}

Keywords-5G, Mobile and wireless communication, Mobile computing for future, Customer centricity, Brand positioning, customer segmentation ****.

\section{INTRODUCTION}

A new mobile generation has appeared approximately every 10th year since the first $1 \mathrm{G}$ system, Nordic Mobile Telephone, was introduced in 1981. The first $2 \mathrm{G}$ system started to roll out in 1991, the first 3G system first appeared in 2001 and 4G systems fully compliant with IMT Advanced were standardized in 2012. The development of the 2G (GSM) and 3G (IMT-2000 and UMTS) standards took about 10 years from the official start of the R\&D projects, and development of 4G systems started in 2001 or 2002.[1][2] Predecessor technologies have occurred on the market a few years before the new mobile generation, for example the pre-3G system CdmaOne/IS95 in the US in 1995, and the pre-4G systems Mobile WiMAX in SouthKorea 2006, and first release-LTE in Scandinavia 2009. In April 2008, NASA partnered with Geoff Brown and Machine-to-Machine Intelligence (M2Mi) Corp to develop $5 \mathrm{G}$ communications technology [3]. Table 1 shows the evolution of mobile technology.

Table 1

\begin{tabular}{|l|l|l|}
\hline \multirow{2}{*}{$\begin{array}{l}\text { Genera } \\
\text { tion }\end{array}$} & Evolution of Technology Generation \\
\cline { 2 - 3 } & Primary Services & $\begin{array}{l}\text { Key } \\
\text { Differentiator }\end{array}$ \\
\hline $1 \mathrm{G}$ & Analogue phone calls & Mobility \\
\hline $2 \mathrm{G}$ & $\begin{array}{l}\text { Digital phone calls and } \\
\text { messaging }\end{array}$ & $\begin{array}{l}\text { Secure, mass } \\
\text { adoption }\end{array}$ \\
\hline $3 \mathrm{G}$ & $\begin{array}{l}\text { Phone calls, messaging, } \\
\text { data }\end{array}$ & $\begin{array}{l}\text { Better internet } \\
\text { experience }\end{array}$ \\
\hline
\end{tabular}

\begin{tabular}{|c|c|c|}
\hline \multirow{2}{*}{$\begin{array}{l}\text { Genera } \\
\text { tion }\end{array}$} & \multicolumn{2}{|c|}{ Evolution of Technology Generation } \\
\hline & Primary Services & $\begin{array}{l}\text { Key } \\
\text { Differentiator }\end{array}$ \\
\hline $3.5 \mathrm{G}$ & $\begin{array}{l}\text { Phone calls, messaging, } \\
\text { broadband data }\end{array}$ & $\begin{array}{l}\text { Broadband } \\
\text { internet, } \\
\text { applications }\end{array}$ \\
\hline $4 \mathrm{G}$ & $\begin{array}{l}\text { All-IP services (including } \\
\text { voice, messaging) }\end{array}$ & $\begin{array}{l}\text { Faster } \\
\text { broadband } \\
\text { internet, lower } \\
\text { latency }\end{array}$ \\
\hline
\end{tabular}

$5 \mathrm{G}$ (5th generation mobile networks or wireless systems) denotes the next major phase of mobile telecommunications standards beyond the current 4G/IMT-Advanced standards. NGMN Alliance or Next Generation Mobile Networks Alliance defines 5G network requirements as:

- $\quad 1-10 \mathrm{Gbps}$ connections to end points in the field (i.e. not theoretical maximum)

- 1 millisecond end-to-end round trip delay (latency)

- $1000 x$ bandwidth per unit area

- 10-100x number of connected devices

- (Perception of) $99.999 \%$ availability

- (Perception of) $100 \%$ coverage

- $\quad 90 \%$ reduction in network energy usage

- Up to ten year battery life for low power, machinetype devices

Next Generation Mobile Networks Alliance feel that 5G should be rolled out by 2020 to meet business and consumer demands.[4] In addition to simply providing faster speeds, 
they predict that $5 \mathrm{G}$ networks will also need to meet the needs of new use-cases such as the Internet of Things as well as broadcast-like services and lifeline communications in times of natural disaster. We will see self-driven cars where they interact with 50-100 other cars in the locality. Millions of devices will be able to talk to themselves. In order to meet these demands, $5 \mathrm{G}$ networks will need to adopt new technologies such as mesh networking, whereby devices communicate with each other directly rather than relying on network operators' base stations. This will increase the bandwidth available, lower power consumption, reduce infrastructure costs, improve spectral efficiency and increase the resilience of the network, but could also lead to higher latencies.[5][6][7]

Advances in technology and communication, combined with the explosive growth in data and information, have given rise to a more empowered global consumer.[8] Consumers are connected with the world and there is a lot of opportunity for customers to either complain or rave about you. The business who respond best to what customers want now are most likely to succeed. Businesses have to meet the customer requirements in the real time. A clear understanding of customer needs and behaviors across the organization will help drive profitable growth strategies and provide the confidence to invest in opportunities at a time when staying within budget is extremely difficult.

In this paper, I am going to study how the advancement in mobile technology will impact customer engagement and propose a model that can guide the businesses to address their customer in the right way.

\section{OBJECTIVES OF THIS STUDY}

Technology is growing at an exponential rate, influencing consumer behavior and customer strategies. By 2020, $80 \%$ of the global population will have access to mobile telephony and more than $60 \%$ to smartphones or low-cost tablet computers. It is anticipated there will be more than 50 billion connected devices globally, with mobile being the primary internet device for most individuals.[8] A $2 \%$ increase in customer retention has the same effect as decreasing cost by $10 \%$. It's easier to make your customer to buy $10 \%$ more than get a $10 \%$ new customer base. Figure 1 shows some of the social media statistics and this is only going to increase.

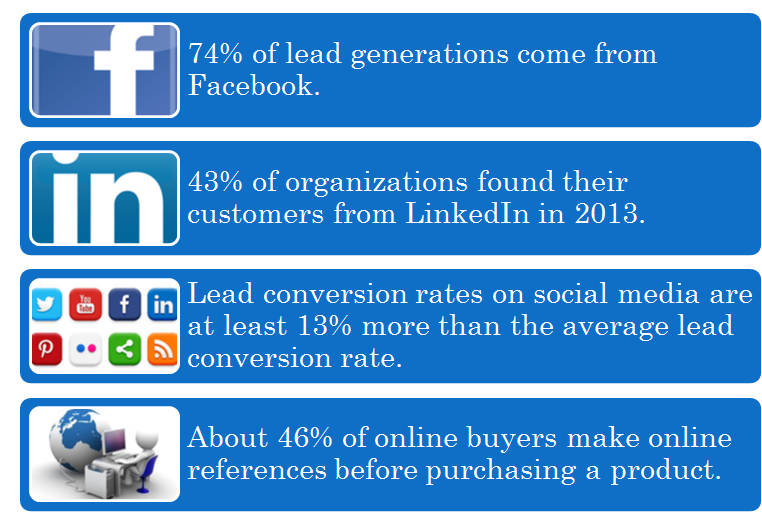

Fig 1: Social Media Statistics

The purpose of this study is to take a step toward understanding what ' $5 \mathrm{G}$ ' really means in the technological sense and the impact it can have on the customer service. I have used publically available data to understand the technological changes the impact it can have on customer centricity approach by the businesses. I am using case study method to arrive at my findings. I will also extend the findings to propose a framework that can guide businesses to take a complete new approach their customer centricity strategy in the new world.

My findings are based on reading various research papers and technical papers published over last 5 years. I have visited websites of the Fortune 100 companies to understand their customer strategies and this paper is based on result of the study. The paper talks about the revolution that will be created by $5 \mathrm{G}$ and the businesses and the solution providers should be ready to use them to their benefit. This paper also opens up many frontiers for future research in the related areas.

In summary, there are three key take away from this study: 1. What is $5 \mathrm{G}$ and the impact it can have on consumer behavior

2. Developing a real time customer strategy

3. Designing a Customer Centric framework.

\section{5G AND IMPACT ON CONSUMER BEHAVIOR}

In new $5 \mathrm{G}$ world, where internet will be fast and accessible (both in terms of connectivity and cost), customers will be more connected and will always be available online. There will be millions of devices connected and talking to each other. As shown in figure 2, there are already 2.7 million smart phones, this will increase many folds by the time $5 \mathrm{G}$ will be made available to public. The challenge of the businesses will be how to transition from existing product and traditional distribution models to those that deliver what customers want. Customers prefer products to be simpler and more transparent, so it is easier to make informed choices. Recognizing the value of the customer relationship is vital - at the point of initial sale and over the customer lifetime. In this section, we will look at the top 5 factors that came out from my study on how businesses will get customer insights and tailor their offerings on individual customer rather than group or geographies.

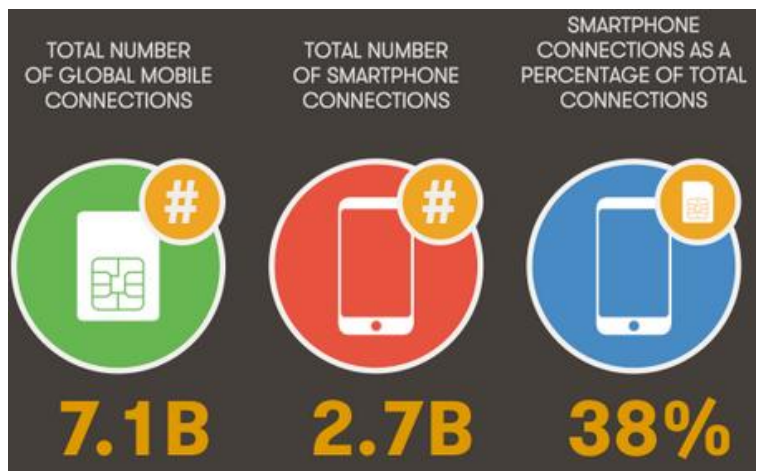


Fig 2: Mobile connections by device

\subsection{Segmentation on Individual Customer}

Till now most of the businesses create customer segments as group of customers based on geographies, demographics, etc. 5G will make identification and tracking of individual customers possible. This will help customers to get insights about their individual customers and offer they tailored services and products. This will increase customer satisfaction and lead to customer loyalty.

\subsection{Customized Pricing}

$5 \mathrm{G}$ technologies will help businesses to offer Omni channel experiences to their customers, which means, irrespective of the channel (physical, online, phone, etc) customers will get the same type of experience while buying the products. Businesses will also be able to recognize their customers uniquely and will reward their loyalty. One way to reward the loyal customers will be to offer personalized pricing. The price and the discount will be offered will be based on the individual customer and their past history unlike in current time where

\subsection{Customer Sentiment Analysis}

Social media analytics will play more important role on how businesses will sell to their customers. Twitter is analyzing tweets of its users to understand what kind of person she or he is. $5 \mathrm{G}$ will be able to analyze customers in real time and businesses will be able to predict the future buying pattern of their customers.

\subsection{Redefine Customer Relationships}

5G technology will lead to businesses to deliver solutions that pushing their products. Businesses will be able to understand the needs of the customer better and offer personalized customer service. For example, marketing messages and emails are currently sent to a group of customers but with better knowledge of each customers, more customized messages will be sent to the customer resulting in better conversion rate and increase in sales. Future successful businesses will be nimble and able to respond quickly and effectively to changing market conditions, new consumer demands and emerging regulations.

\subsection{Differentiate Your Organization}

I also found that most of the Fortune 100 organizations are aligning and redefining their strategy for the future. Organizations have customer centric strategy with other strategies and people inside the organization internally aligned, and everyone should have a sense of where the company is going. Day-to-day decisions should be aligned with that direction. A culture of innovation keeping customer at the center needs to be developed. As Henry Ford had said, salaries are not paid by the organizations but customers pay the salary.

\section{REAL TIME CUSTOMER STRATEGY}

$5 \mathrm{G}$ is an enabler of the fully Connected Network Society. Enabler of the World where there is unlimited access to information and sharing of data ANYTIME to ANYWHERE to ANYONE \& ANYTHING. Its not just about broadband but about any kind of connectivity. In this section, I have studied the various strategies that can differentiate in a $5 \mathrm{G}$ world.

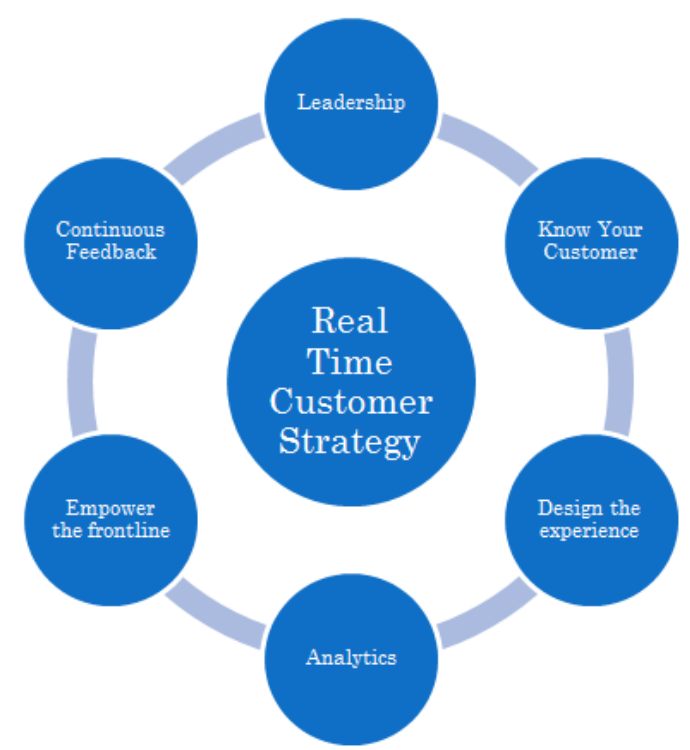

Fig 3: Real-time customer strategy

\subsection{Leadership}

$5 \mathrm{G}$ gives the opportunity for brands to be smarter and more contextually relevant when tracking the customer journey. In a 5G world, brands will be able to multi-hop across consumers' various environments with ease via mobile, device-to-device discovery. Communication will increase tenfold, meaning devices will make real sentences. With so much of opportunity available, one has to be a leader in adopting this technology. Leadership will require companies to create new services and new experiences based on all the different ways one can interact and connect with each other across sensors and devices. Some areas where leadership position will be required are: Scalability \& Adaptability, User-Centric Design and Unified Platform.

\subsection{Know Your Customer}

In a $5 \mathrm{G}$ world, brands will be able to multi hop across consumers' various environments with ease via mobile, device-to-device discovery. You will never have to predict a customer journey again; you will know it in real time. World is going to be a connected place breaking the boundary of developed and developing nations. Internet speed in a high end hotel in Nigeria is same as the speed offered by any high end hotel in USA, which shows the globe has become one connected place and businesses can't ignore any customer of theirs. 


\subsection{Design the Experience}

Things are moving for real and we don't have to wait till 2020 to see the changes. A BSH home appliance manufacturer developed cameras inside its latest line of refrigerators for food tracking purpose. A photo is sent on your phone so that you know what's in your fridge on your way home. A CPG brand was able to come in and dish out recipes based on the context of the items in your fridge. You have smart suitcases and self-driven cars which cannot be practically used right now but concept are already designed and tested which makes us ready for future.

\subsection{Analytics}

Combining the power of physics based analytics, predictive algorithms, and deep domain expertise will help to design solutions of the future. Big data analytics, intelligent machines are some of the areas which will be helped by $5 \mathrm{G}$ technologies.

\subsection{Empower the Front Line}

Mobile prediction propositions are only getting smarter and better. Let's say your heating unit is failing based on the changes predicted by your thermostat. The heating unit company is already pinged and has already ordered the replacement part. As its being shipped, a technician has been notified- on their mobile device-of the problem and the arrival of the item. All these are done seamlessly. Another example, where sales associate in a store is able to take a decision on the price that can be offered to a customer. All these are example of empowering the front line executives.

\subsection{Continuous Feedback}

When you start to look back over time, there are a ton of ideas that failed in the digital and mobile space. Some of them could have been just an issue of wrong place, wrong time. We need to learn from our past and use that knowledge to build for future.

\section{COSTOMER CENTRIC FRAMEWORK}

Based on my study I found that all the businesses can be classified into 5 stages: Underdeveloped, Emerging, Developing, Advancing and Leading. The aim of all the businesses is to reach the Leading stage but not all are able to reach to that stage. Companies in Leading stage offer more than what is needed to just be in business and are referred to as "Exciters" and "Delighters" as they offer much better service and products to their customers. Customers' satisfaction and customer loyalty level is much higher with an organization in leading stage. My framework where I talk about how an organization can move from Underdevelop stage to Leading stage is shown in figure 4.

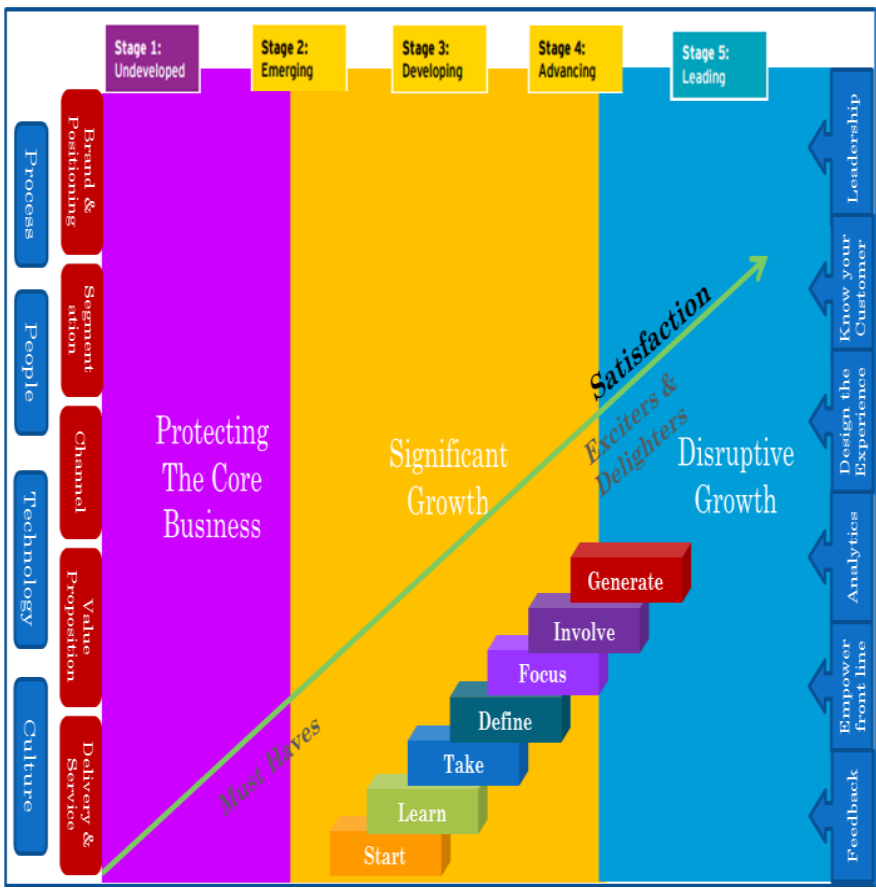

Fig 4: Customer Centric Framework

There are two types of levers than can enable a journey from stage 1 to stage 5 . I found that its crucial and must of the organizations to have level 1 levers which are - People, Processes, Technology and Culture, oriented towards providing a customer centric strategy. The leader of the organization must talk and practice customer centricity and get whole organization oriented towards that. For example, in Stage 1 organization (People), associates do not own a customer, compensation is not based on customer satisfaction level, the customer facing associates do not have enough support from back office team and they lack authority to take decision to resolve customer issues. In Stage 5 organization, Customer-centric culture pervasive at all levels of the organization and associates take clear ownership for customer problems and manage issues through to resolution

Once the organization has the basic level 1 levers in place, they look to organize level 2 levers. The Level 2 Levers are: Brand Positioning: Brand positioning has to be distinctive to each individual.

Segmentation: Gone are the days of identifying customer segments as "single women 18-35" or "married couples with children under 2.". 5G will help in taking account each individual

Selling channel: It's going to be an Omni channel world.

Value Proposition: Compelling value proposition will only excite the customers and 5G will help in tailored solutions by understanding customer requirement and not about pushing their products.

Delivery: Delivery will be done where customer wants and how they want. 
Service: Customer service will be an ongoing focus and not wait for the customer to call you.

I propose a 7 steps design that can take a stage 1 business to the stage 5. These are:

Start: Start to build a culture that puts the customer at the center and aligns objectives, targets, rewards and recognition with customer needs

Learn: Learn to be adaptive-use detailed customer insight to test, learn and act quickly, accelerating the process with prototypes

Take: Take a realistic look at your propositions (solutions, not products) — what will it take to deliver what customers want at a price they want to pay and still make money?

Define: Define your target customers and their needs

Focus: Focus on some key levers and build momentum throughout the organization

Involve: Involve distributors and, together, build stronger partnerships

Generate: Generate customer engagement across their life cycle - this is vital to building loyalty.

I have designed this framework where a business can first analyze where they are place. Then decide on the destination stage where they want to move and lastly put all the levers in place. This framework will help the companies to benchmark itself with its peers from the industry and also help in designing a strategy to move forward.

\section{CONCLUSION}

The journey to customer centricity where an organization can move from Underdeveloped stage to Leading stage will not be an easy endeavor. In building a strong foundation for the future, businesses will need to focus on a new set of core principles to redefine relationships while taking advantage of emerging $5 \mathrm{G}$ technologies. There will be many challenges in developing the critical new capabilities but $5 \mathrm{G}$ offers exciting new opportunities that offer practical steps for the businesses to consider along the way as they build momentum for customer centricity across their organizations.

\section{REFERENCES}

[1] Akhtar, Shakil, "2G-5G Networks: Evolution of Technologies, Standards, and Deployment", Hershey, Pennsylvania, United States: IGI Global. pp. 522532, 2005

[2] a US Department of Homeland Security program, "Emerging Wireless Technologies; A look into the future of wireless communications - beyond 3G", SafeCom, 2011
[3] Michael Curie, Michael Mewhinney, Sarah Cooper, "NASA Ames Partners With M2MI For Small Satellite Development", Nasa.gov, April 2008

[4] NGMN Alliance, "5G White Paper - Executive Version", NGMN.org, Dec 2014

[5] Ian Poole, "5G Mobile / Cellular Technology", radioelectronics.com, 2015

[6] NGMN Alliance, "5G White Paper ", NGMN.org, Feb 2015

[7] Harpreet S. Dhillon, Giuseppe Caire, "Scalability of Line-of-Sight Massive MIMO Mesh Networks for Wireless Backhaul", Virginia Tech, 2014

[8] Ernst \& Young, "The journey toward greater customer centricity", 2014

[9] GSMA Intelligence, "Understanding 5G: Perspectives on future technological advancements in mobile", Dec 2014

[10] Oglivy \& Mather, "Mobile World Congress 2015", March 2015

[11] Abinash Tripathy, "How mobile devices are forcing businesses to rethink customer service", venturebeat.com, March 2013

[12] Matthew D’Uva , Jason Rosser, "Mobile Technology: Changing the Landscape of Customer Care", consumeraffairs.com, March 2015

[13] Brenda Priddy, "4 Ways to Use New Technology for Better Customer Service", salesforce.com, Oct 2013 